\title{
Thermal Tachyon Cosmology
}

\author{
Yun-Song $\mathrm{Piao}^{a, c}$ and Yuan-Zhong Zhang ${ }^{b, c}$ \\ ${ }^{a}$ College of Physical Sciences, Graduate School of Chinese Academy of Sciences, \\ YuQuan Road 19A, Beijing 100049, China \\ ${ }^{b}$ CCAST (World Lab.), P.O. Box 8730, Beijing 100080 and \\ ${ }^{c}$ Institute of Theoretical Physics, Chinese Academy of Sciences, \\ P.O. Box 2735, Beijing 100080, China
}

\begin{abstract}
We show that in a multi $\mathrm{D} \overline{\mathrm{D}}$ branes system with high temperature, there may exist a thermal cosmological phase before usual tachyon inflation. Though this thermal phase can be very transitory, it may has some interesting applications for early tachyon/brane cosmology.

PACS numbers: 98.80.Cq
\end{abstract}


Recently many efforts have been devoted to studying non-BPS configurations of branes, such as DD̄ brane pairs, non-BPS branes [1, 2, 3, 4]. For such a configuration, the spectrum of open strings will contain a tachyon field, for non-BPS brane it is real and for DD brane pairs it is complex, which indicates that this configuration is unstable. The tachyon field will roll down from the vacuum of open string toward the minimum of the tachyon effective potential. There has been a lot of studies on various cosmological effects of the rolling tachyon [5, 6, 7, 8, ]. .

However, for generic initial conditions, an early universe with more high temperature may be more possible. Thus it is interesting to investigate the cosmology of this kind of non-BPS configuration in high temperature. It has been shown that in high temperature the non-BPS configuration can become stable and not decay [10, 11, 12]. Thus in analogy with high temperature symmetry restoration in usual field theories [13], see [14] for a thermal inflation, there may be a thermal cosmological evolutive phase before the usual tachyon inflation, especially when the number of branes is very large. The interest of this thermal evolution lies in providing an initial condition for subsequent tachyon inflation. We will show and discuss it in the following.

We focus on a system of N D3-D̄3 branes pairs with finite temperature. The free energy for the N D3-D̄3 branes and mixed gas system at temperature $T=\beta^{-1}$ can be written as, following Ref. [10],

$$
\begin{aligned}
f(\phi, \beta) & =2 \tau_{3}\left(\operatorname{Tr} e^{-2|t|^{2}}\right) \\
& +\frac{c N^{2} \Omega}{(2 \pi)^{3} \beta^{4}} \int_{0}^{\infty} x^{2} \ln \left(1-e^{-\sqrt{x^{2}+\beta^{2} m^{2}}}\right) d x
\end{aligned}
$$

where

$$
\tau_{3}=\frac{M_{s}^{4}}{(2 \pi)^{3} g_{s}}
$$

is D3 or D 3 brane tension, $t$ is related to the background tachyon field $\phi$ appearing in the D brane worldsheet action through an error function as follows

$$
|\phi|=\sqrt{\frac{\pi}{2}} \operatorname{Erf}(|t|)
$$

and $\Omega$ is the volume of a unit two-sphere, the constant $c$ is given by $c=8+8(7 / 8)$ for the relevant eight bosonic and eight fermionic degrees of freedom, which include gauge fields, transverse scalars and superpartners and form a mixed gas system, $m \sim M_{s}|\phi|$ is the mass 
given to gauge fields by Higgs effect. If the tachyon fields start at $\phi=0$, then the tachyon rolling along the effective potential will lower the energy of the system, but it also gives mass to the relative gauge fields and decrease the entropy of the mixed gas system. Thus, an amount $\delta \phi$ gives mass to $N$ out of the $N^{2}$ species of particles in the gas. Varying (II), one have

$$
\delta f(\phi, \beta)=-2 \tau_{3}(\delta \phi)^{2}+\frac{15}{24} N \beta^{-2}(\delta m)^{2}
$$

Therefore, for large enough temperature

$$
T \geq T_{c}=\frac{T_{h}}{\sqrt{g_{s} N}}
$$

where the numerical constants have been disregarded, and $T_{c}$ is regarded as a critical temperature, $\delta f>0$, i.e. the open string vacuum becomes a minimum of the free energy and is stable. In this case, the tachyon field is no longer tachyonic and the D $\bar{D}$ brane pairs do not annihilate. $T_{h} \sim M_{s}$ is the Hagedorn temperature, for open string, which is a limiting temperature, i.e. in all senses $T<T_{h}$. When approaching this temperature, the energy is converted to the massive modes of open string and the creation of D $\bar{D}$ brane pairs may become more important [15]. Since the existence of the limiting temperature, from (51), we see that in usual case, $T$ can hardly exceed $T_{c}$, only when $g_{s} N \gg 1, T>T_{c}$ becomes possible. But in this case, open strings are strongly coupled, which leads that the perturbative calculation used here becomes distrusted. However we may take $g_{s} N \sim \mathcal{O}(1)$ marginally for our purpose. In this case $T_{c} \sim T_{h}$. Thus when the temperature $T \sim T_{h}$, the system will be stable. Further to suppress closed string loops, $g_{s} \ll 1$ must be taken, which makes the radiation process of closed string disfavored, and thus the temperature of system is mainly relevant to open string. Thus a large enough $N$ will be generally required, which may be also required by some other cosmological consideration [8, 9, 16, 17].

Now we assume that $N \gg 1$ and $T_{c} \sim T_{h}$ and focus on the cosmology of brane system consisting of $N$ DD brane pairs. In this case, the open string vacuum is stable. The energy density of mixed gas system considered is given by

$$
\rho_{\text {gas }} \sim N^{2} T^{4}
$$

since the number of massless mode of open strings on $N$ DD branes pairs is proportional to $N^{2}$. Thus initially for $T \sim T_{c} \sim M_{s}$, we have $\rho_{g a s} \sim N^{2} M_{s}^{4}$, in the meantime the energy 
density of branes tensor is $\rho_{\text {brane }} \sim N \tau_{3}$. Thus considering (2), we have

$$
\rho_{\text {brane }} \sim \frac{N M_{s}^{4}}{(2 \pi)^{3} g_{s}} \sim \frac{N^{2} M_{s}^{4}}{(2 \pi)^{3}} \sim 10^{-2} \rho_{\text {gas }},
$$

where $g_{s} N \sim \mathcal{O}(1)$ has been used. This result indicates that when the temperature of the brane system approaches the Hagedorn temperature $T_{h}$, the energy density of mixed gas system will be dominated, while the branes tension is subdominated. Thus generally there will be a radiation-dominated thermal phase in the tachyon/brane cosmology before the tachyon becomes tachyonic. During this period,

$$
H^{2} \sim \frac{N^{2} M_{s}^{4}}{M_{p}^{2}}=\frac{g_{s}^{2} N^{2}}{v} M_{s}^{2},
$$

where in second equation, we use $M_{p}^{2}=\frac{v M_{s}^{2}}{g_{s}^{2}}$, where $v=\left(M_{s} R\right)^{6} \gg 1$ is the volume of extra spaces in string unit. Thus for $g_{s} N \sim \mathcal{O}(1)$, we have $\frac{1}{H} \gg l_{s}$, where $l_{s}=\frac{1}{M_{s}}$ is the string length. Therefore, during the cosmological evolution of brane system, the description of 4D effective field theory for tachyon/brane cosmology seems reasonable.

In the following we will use the $4 \mathrm{D}$ effective description for the cosmology of above brane system. Initially the temperature $T \sim T_{c} \sim T_{h}$, and the brane cosmology is dominated by the mixed gas system with the energy density $\rho \sim 1 / a^{4}$ where $a$ is the scale factor of expanding brane cosmology. In this case the temperature $T \propto \frac{1}{a}$, and thus will decrease rapidly with the cosmological expansion. When the temperature drops to $T \sim 0.3 T_{h}$, in which $\rho_{\text {gas }} \simeq \rho_{\text {brane }}$, the energy density of branes tensor will begin to dominated the universe. This means that

$$
H^{2} \simeq \frac{2 N \tau_{3}}{M_{p}^{2}},
$$

and is a nearly constant. Thus the universe will enter into an inflation stage, as usual tachyon inflation. In this stage, since $T \leq T_{h} \sim T_{c}$ the open string vacuum has became unstable and will evolve toward closed vacuum, i.e. the minimum of tachyon potential. The relevant cosmology with large $N$ branes has been discussed in Ref. 8, 9].

Note also that during the radiation-dominated, $\rho \sim 1 / t^{2}$. Thus the time of existence of this thermal phase is given by

$$
t \sim\left(T_{h}^{2} / T^{2}\right) t_{c} \sim 10 t_{c}
$$

which seems very transitory. However, the transitory appearance of this thermal evolutive phase before usual tachyon inflation may be interesting for some purposes as follows, Imagining a model where branes warp some cycles of the compact manifold, and the volume 
of cycles is generally not constant due to the pinching singularities of compact manifold, we can see that branes and antibranes will minimize their energy and move towards points in which the cycles have minimal volume [19]. The kind of singularities or points can be regarded as collectors of branes and antibranes, in which branes will be collected and coincident. The occurrence of thermal expanding phase will be helpful to remove singularities or points of small cycle volume in generic compact manifolds and prevent early collapse of brane universe, In addition, since in $T \sim T_{h} \sim T_{c}$ the open string vacuum is a minimum and is stable again decay, it is easily localized $\phi$ into $\phi=0$ from the viewpoint of effective field theory. This in some sense corresponds to provide an initial conditions for subsequent tachyon inflation.

In summary, we discuss the cosmological evolution of a multi DD branes system with high temperature, and its possible application for early universe. This work implies that the brane cosmology with high temperature may have some interesting phenomena, which is worth studying further.

Acknowledgments The author would like to thank Qing-Guo Huang and Miao Li for earlier helpful discussions. This work is supported in part by NNSFC under Grant Nos: 10405029 and 90403032, as well as in part by National Basic Research Program of China under Grant No: 2003CB716300.

[1] A. Sen, JHEP 9808 (1998) 012, hep-th/9805170 Int. J. Mod. Phys. A14 (1999) 4061, hep-th/9902105; hep-th/9904207; JHEP 9912 (1999) 027, hep-th/9911116

[2] A. Sen and B. Zwiebach, JHEP 0003 (2000) 002, hep-th/9912249, V.A. Kostelecky and S. Samuel, Nucl. Phys. B336 (1990) 263; N. Loeller and W. Taylor, Nucl. Phys. B583 (2000) 105, hep-th/0002237; W. Taylor, hep-th/0208149.

[3] E. Witten, Phys. Rev. D46 (1992) 5467, hep-th/9208027; Phys. Rev. D47 (1993) 3405, hep-th/9210065 S.L. Shatashvili, Phys. Lett. B311 (1993) 83, hep-th/9303143 hep-th/9311177; J.A. Minahan and B. Zwiebach, JHEP 0103 (2001) 038, hep-th/0009246 D. Kutasov, M. Marino and G. Moore, hep-th/0010108.

[4] A. Sen, hep-th/0203211; hep-th/0203265.

[5] G. Gibbons, hep-th/0204008; S. Mukohyama, hep-th/0204084 A. Sen, hep-th/0204143. 
A. Feinstein, hep-th/0204140; T. Padmanabhan, hep-yh/0204150 G. Shiu and I. Wasserman, hep-th/0205003 T. Padmanabhan and T.R. Choudhury, hep-th/0205055. S. Mukohyama, hep-th/0208094 A. Sen, hep-th/0209122 C. Kim, H.B. Kim and Y. Kim, hep-th/0210101; H. Lee and W.S. l'Yi, hep-th/0210221; J.S. Bagla, H.K. Jassal, T. Padmanabhan, astro-ph/0212198 M. Sami, P. Chingangbam, T. Qureshi, hep-th/0301140 C. Kim, H. B. Kim, Y. Kim, O-K. Kwon, hep-th/0301142; S.-J. Rey, S. Sugimoto, hep-th/0303133; H.K. Jassal, astro-ph/0303406, M. Sami, N. Dadhich, T. Shiromizu, hep-th/0304187.

[6] L. Kofman and A. Linde, hep-th/0205121.

[7] M. Fairbairn and M.H. Tytgat hep-th/0204070 D. Choudhury, D. Ghoshal, D.P. Jatkar and S. Panda, hep-th/0204204 M. Sami, hep-th/0205146 M. Sami, P. Chingangbam, T. Qureshi, hep-th/0205179 Y.S. Piao, Q.G. Huang, X.M. Zhang and Y.Z. Zhang, hep-ph/0212219; Z.K. Guo, Y.S. Piao, R.G. Cai and Y.Z. Zhang, hep-ph/0304236.

[8] Y.S. Piao, R.G. Cai, X.M. Zhang and Y.Z. Zhang, Phys. Rev. D66 (2002) 121301, hep-ph/0207143

[9] M. Majumdar and A. Christine-Davis, hep-th/0304226.

[10] U.H. Danielsson, A. Guijosa and M. Kruczenski, hep-th/0106201,

[11] W. Huang, hep-th/0106002, hep-th/0211127;

[12] K. Hotta, hep-th/0212063 hep-th/0303236.

[13] L. Dolan and R. Jackiw, Phys. Rev. D9 (1974) 3320.

[14] D.H. Lyth and E.D. Stewart, Phys. Rev. D53 (1996) 1784, hep-ph/9510204.

[15] S.A. Abel, J.L. Barbon, I.I. Kogan and E. Rabinovici, JHEP 9904 (1999) 015, hep-th/9902058 J.L. Barbon, I.I. Kogan and E. Rabinovici, Nucl. Phys. B544 104 (1999), hep-th/9809033.

[16] S.H. Alexander, R.H. Brandenberger and D. Easson, Phys. Rev. D62 (2000) 103509, hep-th/0005212,

[17] M. Majumdar and A. Christine-Davis, hep-th/0202148, hep-th/0304153.

[18] S.H. Alexander, Phys. Rev. D65 (2002) 023507, hep-th/0105032, S.H. Alexander, R.H. Brandenberger and M. Rozali, hep-th/0302160.

[19] R. Easther, B.R. Greene and M.G. Jackson, hep-th/0204099. 